vor allem darauf abzustellen, welche Ziele die eine Versammlung anmeldenden Personen, ihr Umfeld, die angeworbenen Teilnehmer, ihre organisatorischen Träger einschließlich der mit ihnen netzwerkartig verbundenen Personen, Organisationen verfolgen, welcher grundlegenden Weltanschauung die Veranstaltung oder ihre prospektiven Teilnehmer verpflichtet sind. Insoweit geht es nicht an, die sich aus der beabsichtigten Verwendung von Symbolen, einer uniformierten, uniformähnlichen Kleidung, dem Einsatz von Trommeln oder Fahnen ergebenden Ziele zu ignorieren. Solche Umstände sind vielmehr klare Indizien für die wahren Ziele einer Versammlung. Deshalb kann hier nicht mit dem Grundsatz der Verhältnismäßigkeit ordnungsrechtlichen Einschreitens und dem Vorrang von Auflagen argumentiert werden. Die Beseitigung der äußeren Kennzeichen für die wahren Ziele einer sich gegen den Gedanken der Völkerverständigung richtenden Versammlung kann an deren Grundlinie nichts ändern und deshalb deren Unfriedlichkeit nicht in eine verfassungskonforme Friedlichkeit verwandeln. Allenfalls dienen solche Auflagen der Maskierung der wahren Absichten und Ziele, erleichtern also letztlich deren Verfolgung, anstatt den Auftrag zu erfüllen, den öffentlichen Frieden wirksam zu schützen.

\title{
Fredrik Roggan Am deutschen Rechtswesen soll die Welt genesen? Eine rechtspolitische Skizze zum Urteil des BGH vom I 2. I 2. 2000
}

Stellt ein Ausländer von ihm verfasste Äußerungen, die den Tatbestand der Volksverhetzung i.S. des $\rrbracket_{130}$ I StGB erfüllen ("Auschwitz-Lüge ), auf einem ausländischen Server in das Internet, der Internetnutzern in Deutschland zugänglich ist, so tritt ein zum Tatbestand gebörender Erfolg $(\mathbb{9}$ I Alt. 3 StGB) im Inland ein, wenn diese Äußerungen konkret zur Friedensstörung im Inland geeignet sind. BGH, Urt. v. 12.12.2000-1 StR 184/00 (LG Mannheim), NJW 2001, $624 \mathrm{ff}$.

1. Es bedarf keiner näheren Erläuterung, daß die Auschwitzlüge und ihr vergleichbare Äußerungen den Tatbestand der Volksverhetzung erfüllen. Gewiß ist die besprochene BGH-Entscheidung unter diesem Gesichtspunkt unproblematisch, und man ist angesichts der rechtsextremistischen Umtriebe, über die man nahezu täglich in den Zeitungen lesen kann, versucht, das Ergebnis des BGH auch zusammen mit seinen Entscheidungsgründen zu begrüßen. Allzu leicht möchte man sich der spontanen Reaktion, daß es in diesem Fall ja den »Richtigen « (einen Rechtsextremisten) getroffen hat, hingeben. Und gleichzeitig könnte man als Ergebnis der Entscheidung konstatieren, daß es in dem immer bedeutungsvolleren Bereich der Strafbarkeit von Äußerungen im Internet keine Strafbarkeitslücke gibt. Indessen sind die Gründe des $\mathrm{BGH}$ keineswegs so unproblematisch, wie es auf den ersten Blick den Anschein haben könnte.

2. Der BGH hatte mit seiner Entscheidung zwangsläufig juristisches Neuland zu betreten, was sich vorliegend insbesondere aus dem Umstand ergab, daß sich im zugrunde liegenden Fall überhaupt nur mühsam ein Inlands-Bezug herstellen ließ. ${ }^{\mathrm{T}}$

I U.a. deshalb erscheint es wenig verwunderlich, daß selbst Tageszeitungen das Urteil z. T. sehr ausführlich besprachen, vgl. etwa Berliner Zeitung v. I3. 12. 2000 , S. 17. 
Die fraglichen Inhalte wurden nämlich nicht von einem Deutschen ( $\$ 7 \mathrm{II} \mathrm{StGB}$ ) in das Internet eingestellt und auch nicht auf einem deutschen Server abgelegt oder sonst von Deutschland aus verbreitet ( $\$ 3$ StGB: Tatort im Inland). Bei solchen Konstellationen wäre die Zuständigkeit der deutschen Gerichtsbarkeit vergleichsweise unproblematisch festzustellen gewesen. ${ }^{2}$ Vorliegend bedurfte es aber einigen argumentativen Aufwands, um überhaupt eine Strafbarkeit auf dem Territorium der BRD zu begründen. Die Entscheidung betrifft daher im Kern die Frage, unter welchen Umständen ein von einem Ausländer vom Ausland aus in das Internet gestellter volksverhetzender Inhalt einen »zum Tatbestand gehörenden Erfolg« im Sinne des $\$ 9$ I Var. 3 StGB bewirken kann und somit die Kriterien des Ubiquitätsprinzips und des Territorialitätsprinzips erfüllt. ${ }^{3}$

Problematisch ist insbesondere, daß es sich beim Tatbestand der Volksverhetzung um ein abstraktes Gefährdungsdelikt handelt, von dem das vom BGH festgestellte abstrakt-konkrete Gefährdungsdelikt eine Untergruppe darstellt. ${ }^{4}$ Damit zusammenhängend war die Frage zu beantworten, wodurch ein originär erfolgsunabbängiges Delikt einen »zum Tatbestand gehörenden Erfolg« ( $\$ 9$ I Var. 3 StGB) haben kann. $\mathrm{Zu}$ diesem Zweck mußte sich das Gericht - wieder einmal ${ }^{5}$ - von der Begriffsbildung der allgemeinen Tatbestandslehre trennen. Nach Auffassung des BGH soll zum "Erfolg" i.S. des $\$ 9$ I Var. 3 StGB nämlich jede abstrakt-konkrete Gefährdung genügen, deren Vermeidung Zweck der jeweiligen Strafvorschriften ist. $\mathrm{Zu}$ dieser Orientierung zieht der BGH die vermeintliche ratio legis des $\$ 9$ I StGB heran (Territorialitätsprinzip) .

3. Dieser Auffassung kann keinesfalls gefolgt werden, denn sie relativiert in nicht mehr hinzunehmender Weise Grundsätze, an denen sich das materielle Strafrecht bislang mit guten Gründen orientiert hat: Eine Strafbarkeit von Auslandstaten ohne Rechtsverletzung oder konkrete Gefährdung im Inland sollte grundsätzlich auf diejenigen Fälle beschränkt bleiben, bei denen ein sinnvoller Anknüpfungspunkt im Inland besteht. ${ }^{6}$ Ausprägung davon ist u. a. das Weltrechtsprinzip in $\$ 6 \mathrm{Nr}$. I bis $8 \mathrm{StGB}$ und das Schutzprinzip in $\$ 5$ Nr. $1,2,3 \mathrm{~b}, 4,5 \mathrm{a}, 10-\mathrm{r}_{4} \mathrm{StGB}$. Auf keines dieser Prinzipien konnte sich der BGH berufen, und er unternahm deshalb - konsequenterweise - nicht einmal einen entsprechenden Versuch. $\mathrm{Zu}$ den in den genannten Vorschriften abschließend aufgezählten Tatbeständen zählen neben anderen Delikten auch solche, die unter Gebrauch des Internets begangen werden können. Exemplarisch genannt sei hier die Verbreitung pornographischer Schriften $(\$ r 84$ III, IV $\mathrm{StGB}$ ). Der Tatbestand des $\$$ I $30 \mathrm{StGB}$ indessen wird in den Katalogen nicht genannt. Das läßt sich auch damit erklären, daß etwa die Auschwitzlüge nicht zu denjenigen Taten gehört, die weltweit strafbewehrt sind. ${ }^{7}$ Das Weltrechtsprinzip beschränkt sich aber darauf, daß das inländische Strafrecht nur für diejenigen Taten gelten soll, durch die gemeinsame, in allen "Kulturstaaten ${ }^{8}$ anerkannte Rechtsgüter verletzt werden. ${ }^{9}$ Nur in solchen Fällen soll der Ergreifungsort maßgeblich für die Anwendung des inländischen Strafrechts sein.

Als erster Befund des BGH-Urteils ist somit festzustellen, daß das Weltrechts- und

2 Tolmein in: Hebecker u.a. (Hrsg.), Neue Medienumwelten zwischen Regulierungsprozessen und alltäglicher Aneignung, Frankfurt/M. 1999, S. 154.

3 Ausführlich Jescheck/Weigend, Lehrbuch des Strafrechts - Allg. Teil, München 1996, S. $178 \mathrm{ff}$

${ }_{4}$ Vgl. BGH NJW 1999, 2129 ; Hilgendorf, NJW 1997, 1875; Satzger, NStZ 1998, I 3 f.

5 BGHSt 36, 255 (257).

6 Derksen, NJW I997, 1880.

7 Hilgendorf, NJW 1997, I 874.

8 Der Begriff wurde vom BGH geprägt und ist durchaus als mißverständlich zu bezeichnen.

9 Vgl. dazu nur Schönke/Schröder-Eser, StGB, 2 . Aufl., Vorben $\$ \$ 3-7$, Rdn. 8. 
das Schutzprinzip eine - gesetzlich nicht vorgesehene - Erweiterung durch inländische Rechtsprechung erfahren hat. Oder anders herum: Die Entscheidung widerspricht Grundgedanken des internationalen Strafrechts. ${ }^{10}$

4. Bedenklich an der Entscheidung ist auch, daß eine dem Wortlaut des Gesetzes (hier: Territorialität) widersprechende Fiktion geschaffen wird: Abstrakte Gefährdungsdelikte sollen einen »Erfolg « haben können, der sich im Inland in Form einer abstrakten Gefahr »realisiert«. Gegen eine solch weite Begriffsdehnung gibt es seit langem in der Literatur geäußerte Bedenken: Es wird z. B. eingewandt, daß die Erfolgsklausel insofern einschränkend auszulegen sei, als die deutsche Strafgewalt nur dann gegeben sein könne, wenn der Täter zielgerichtet gerade in Deutschland eine Wirkung seines Handelns im Ausland hervorrufen will. ${ }^{11}$ Hilgendorf etwa erwägt, einen inländischen Erfolgsort im Sinne des $\$ 9$ I Var. 3 StGB nur dann anzunehmen, wenn sich aufgrund objektiver Merkmale der Tathandlung ein »direkter Inlandsbezug « herstellen läßt (Bsp. deutsche Sprache). ${ }^{12}$ Wirklich konsequent ist freilich nur eine restriktive und am Wortlaut des Gesetzes orientierte Auslegung, denn $\$ 9$ I Var. 3 StGB will gerade nicht jede Auswirkung einer Straftat - also z. B. eine lediglich abstrakte Gefährdung - zum Anknüpfungspunkt für eine Strafbarkeit im Inland machen, sondern nur einen zum Tatbestand gehörenden Erfolg, zu dem allenfalls eine konkrete Gefährdung eines Rechtsguts im Einzelfall zu zählen ist. ${ }^{13}$ Das ergibt sich nicht zuletzt aus der Entstehungsgeschichte der Vorschrift, die den Geltungsbereich des deutschen Strafrechts begrenzen wollte: ${ }^{14}$ Es wird seitdem ausdrücklich bestimmt, daß eben nicht jeder-also nicht auch ein über den tatbestandlichen Erfolg hinausgehender möglicher weiterer - Erfolg Bedeutung für die Bestimmung der inländischen Strafbarkeit haben soll. ${ }^{\text {is }}$

$\mathrm{Zu}$ den Tatbeständen, die einen solchen Erfolg voraussetzen, gehören die verschiedenen abstrakten Gefährdungsdelikte nicht. Der BGH ebnet mit seiner Entscheidung also Differenzierungen der Straftatbestände ein, die im Einzelfall (wie hier) eine internationale Strafbarkeit erst begründen. Zwar wird im Schrifttum mitunter der Versuch unternommen, diese Unterschiedlichkeit für hinderlich zu erklären. Ohnehin geraten Verfolgungs- oder Verurteilungs-»Hindernisse« oder auch allgemein rechtsstaatliche Grundsätze, die sich aus dem Wortlaut der Gesetze ergeben, immer dann besonders schnell in Gefahr, wenn sie zu unerwünschten Ergebnissen führen. Dieser Versuchung scheinen neben dem BGH auch Sieber u. a. erlegen zu sein. ${ }^{16}$ Demgegenüber ist in diesem Punkt aber der mittlerweile wohl herrschenden Meinung in der $\mathrm{z}$. T, vom BGH zitierten Literatur und Rechtsprechung zu folgen, die in Fällen von abstrakten Gefährdungsdelikten einen Erfolgsort verneint ${ }^{17}$ und damit das

Io Vgl. dazu Derksen, NJW 1997, 1880 sowie Kienle, Internationales Strafrecht und Straftaten im Internet, Konstanz 1998 (Diss.), S. 50.

I Collardin, CR I995, 62I.

12 NJW $1997,1876 \mathrm{f}$.

13 Satzger, NStZ 1998, I 14 .

I4 Cornils, JZ 1999, 396; Sieber, NJW 1999, 2070 (Fußnote 46).

is Vgl. dazu die Nachweise bei Cornils, NJW I997, 396 (Fußnote 27).

I6 NJW I999, 2069 f.; vgl. auch Jofer, Strafverfolgung im Internet, Frankfurt/M. 1998 (Diss.), S. 108: »...kontraproduktiv, durch die Festlegung auf einen engen Erfolgsbegriff ungewollte Strafbarkeitslükken entstehen zu lassen...k.

17 Vgl. dazu - jeweils mit weiteren Nachweisen - LK-Gribbohm, StGB (Stand: 1. 3- 1 997), \$9 Rdn. 20; Schönke/Schröder-Eser, StGB, $\$ 9$ Rdn. 6; Tröndle/Fischer, StGB, $\$ 9$ Rdn. 3; Tolmein (Fn. 2), S. I 53 ; Cornils, JZ 1999, 396; Hilgendorf, NJW I997, I 875; Satzger, NStZ 1998, I 15 ; Derksen, NJW 1997, I 880; sehr gründlich Römer, Verbreitungs- und Äußerungsdelikte im Internet, Frankfurt/M. 2000 (Diss.), S. 104 bis $I_{4} 6$ und Kienle (Fn. Io), S. 4 I bis 52; a. A. Barton, Multimedia-Strafrecht, Neuwied/Kriftel 1999, S. 14 f.; Martin, ZRP 1992, 20; Heinrich, GA 1999, 72 ff. 

dieser Stelle verwiesen.

Angesichts der folgenden Erwägungen ist die Annahme eines tatbestandlichen Erfolges bei einem abstrakten Gefährdungsdelikt, das nicht auch unter das Weltrechtsprinzip fällt, nicht ohne Brisanz.

5. Das Ergebnis des BGH führt dazu, daß abstrakte Gefährdungsdelikte, die - ohne sonstigen Inlandsbezug! - durch die Verbreitung im Internet begangen werden können, generell der deutschen Gerichtsbarkeit unterfallen. Sämtliche Inhalte des Internet sind damit am Maßstab des deutschen Strafrechts zu messen, weil sie ja immer auch in Deutschland abrufbar sind und damit im Inland eine - tatbestandliche - Gefährdung der genannten Art hervorrufen können. ${ }^{18}$ Das gilt wohlgemerkt auch für Gefährdungen, die ausschließlich in Deutschland zum Tatbestand eines Strafgesetzes gehören. Die deutschen Strafverfolgungsbehörden mutieren auf diese Weise zu einem globalen Kontrolleur des Internets, ${ }^{19}$ ohne daß ihnen z. B. das Weltrechtsprinzip als Rechtfertigung für eine Strafverfolgung von Auslandstaten zur Seite stände. ${ }^{20}$ Auf diese Weise wird ein Strafanspruch statuiert, der losgelöst ist von tatsächlichen - sinnvollen - Anknüpfungspunkten in Deutschland. Die vom BGH angeführte konkrete Eignung zur Friedensstörung reicht dafür jedenfalls nicht aus.

6. Der BGH beschreitet mit seinem aktuellen Frontalangriff auf die Dogmatik des allgemeinen Strafrechts einen auch politisch höchst brisanten Weg. Ausländer müssen nunmehr, bevor sie einen Inhalt vom Ausland her in das Internet stellen, eine mögliche Strafbarkeit in der BRD prüfen. Zwar mag ein solcher Verdacht angesichts der gegenständlichen Verurteilung wegen der Verbreitung der Auschwitzlüge auf den ersten Blick absurd erscheinen; dennoch drängt sich hier auf, daß es sich um eine neue Version des »Am deutschen Wesen soll die Welt genesen « handelt. ${ }^{21}$ Auch umgekehrt wird die Dramatik der (Fehl-)Entscheidung des BGH deutlich: Nach BGH-Grundsätzen wären auch deutsche Staatsangehörige auf der ganzen Welt strafbar, wenn sie sich beispielsweise für eine universelle Geltung von Menschenrechten via Internet einsetzen und bei einem Besuch desjenigen Landes, in dem diese Rechte gerade nicht gelten, wegen eines (abstrakten) Staatsschutzdelikts festgenommen werden. ${ }^{22}$ Deutsche Rechtsanwälte beispielsweise, die über die Verfolgung der Kurden in der Türkei informieren wollen, hätten zukünftig gründlich zu prüfen, was sie auf ihrer Homepage veröffentlichen können, ohne in der Türkei den Tatbestand der »separatistischen Propaganda« zu erfüllen.

Es ist nicht Aufgabe der Rechtsprechung, sich unter Bruch von Grundprinzipien des internationalen Strafrechts am sog. »Aufstand der Anständigen« zu beteiligen.

18 Cornils, JZ 1999, 395.

19 Tolmein (Fn. 2), S. Is3.

20 Von einer "Kollision der Rechtsordnungen « geht auch Barton (Fn. 17), S. 142, aus, ohne freilich aus dieser

Erkenntnis irgendwelche Konsequenzen zu ziehen.

2I Entsprechend Hilgendorf, NJW 1997, 1874 .

22 Vgl. dazu Römer (Fn. I 7), S. 146. 